\title{
Effect of Electron and/or Ion Nonthermality on Dust Acoustic Wave Propagation in a Complex Plasma in Presence of Positively Charged Dust Grains Generated by Secondary Electron Emission Process
}

\author{
Susmita Sarkar ${ }^{1}$, Subrata Bhakta ${ }^{2}$ \\ ${ }^{1}$ Department of Applied Mathematics, University of Calcutta, Kolkata, India \\ ${ }^{2}$ Department of Mathematics, S.A. Jaipuria College, Kolkata, India \\ Email: susmita62@yahoo.co.in, subratabhakta88@gmail.com
}

Received 2 November 2015; accepted 15 January 2016; published 20 January 2016

Copyright (C) 2016 by authors and Scientific Research Publishing Inc.

This work is licensed under the Creative Commons Attribution International License (CC BY).

http://creativecommons.org/licenses/by/4.0/

cC) (i) Open Access

\begin{abstract}
In this paper we have developed a model to study the role of both electron and ion nonthermalities on dust acoustic wave propagation in a complex plasma in presence of positively charged dust grains. Secondary electron emission from dust grains has been considered as the source of positive dust charging. As secondary emission current depends on the flux of primary electrons, nonthermality of primary electrons changes the expression of secondary emission current from that of earlier work where primary electrons were thermal. Expression of nonthermal electron current flowing to the positively charged dust grains and consequently the expression of secondary electron current flowing out of the dust grains have been first time calculated in this paper, whereas the expression for nonthermal ion current flowing to the positively charged dust grains is present in existing literature. Dispersion relation of dust acoustic wave has been derived. From this dispersion relation real frequency and growth rate of the wave have been calculated. Results have been plotted for different strength of nonthermalities of electrons and ions.
\end{abstract}

\section{Keywords}

Complex Plasma, Nonthermality, Positive Dust, Secondary Electron Emission 


\section{Introduction}

Existence of energetic electrons with energies up to $100 \mathrm{keV}$ was observed at and near the Earth's bow shock [1]. The energized electrons are often recognized as "spike" events when observed by a spacecraft transiting the bow shock. Gosling, J.T. et al. (1989) [2] reported that energetic population often possessed a power law tail with spectral index $3-4$, and that the spectrum became harder in the quasi perpendicular region [2]. The primary process for electron acceleration was first considered to be of adiabatic type although it turned out that the adiabatic drift acceleration was not the only process responsible for the production of energetic electrons [3]. A statistical analysis of electron energy spectra at the bow shock was carried out from Geotail observation by Oka et al. (2005) [4]. The origin of the Fe $6.4 \mathrm{keV}$ flurosescence line observed in the X-ray spectrum of Elias 29 was explained with the help of the Emslie et al. (1986) [5] which provided a relationship between the observed 6.4 keV flux and the power law emission of the non thermal electrons [5] [6]. Oka, M. et al. reported a shock crossing event which showed a gradual profile of non thermal electron flux in association with an intensification of precursor whistlers [7].

Nonthermal plasmas are also widely used in many technical applications, including plasma display panels, energy saving lamps, devices for microbial decontamination and ozonisers [8]-[11]. Recently Becker and Loffhagen (2013) [12] derived a set of moment equations for the theoretical description of electrons for modeling of gas discharge plasmas and semiconductor devices [12].

Theoretical investigations of nonthermal plasmas have been considerably important since early nineties. Motivated by the observations of solitary structures with density depletions made by Freza and Viking satellites [13] [14] existence of both compressive and rarefactive solitary waves were theorically established for nonthermally distributed electrons and cold [15] [16] and warm ions [17]. Ion acoustic electrostatic solitary waves in unmagnetized nonthermal plasmas with inertial ion fluids and nonthermally distributed electrons were investigated by Mamun (2000) [18]. Tang and Xue (2004) [19] obtained nonlinear Schrodinger equation to study the instability of oblique modulation of finite amplitude ion-acoustic waves in an unmagnetized plasma consisting of warm adiabatic ions and nonthermal electrons. It was found that the presence of nonthermal electrons significantly changed the domain of modulational instability. Verheest et al. (2012) [20] have investigated the head on collisions of electrostatic solitons in nonthermal plasmas. All the above studies were performed in electron-ion plasmas in absence of dust. Kakati et al. (2011) [21] have shown that presence of dust grains in plasma changes the shape of the electron energy probability function (EEPF). From laboratory observation they have seen that the number of high energy electrons increases with respect to the number of mid energy range electrons in presence of dust grains indicating that addition of dust grains efficiently thermalizes the plasma.

Dusty plasma has become a challenging field of plasma research since early nineties of the last century after detection of dust particles in different satellite observations. In space environment enormous amount of meteoric material condenses into dust particles and is suspended in the Earth's mesosphere between 80 - $100 \mathrm{Km}$. The presence of dust at such a low altitude where the Debye length and the mean free path is small, constitutes a “dusty plasma”, as compared to the “dust in plasma” at higher "satellite orbit” altitudes. The Earth's atmosphere at these low altitudes also presents us with anomalous physical phenomena like noctilucent clouds, polar summer mesospheric echoes (PMSE) and sporadic sodium layers. The occurrence of each of these phenomena has been researched extensively in the literature, and in some or the other way each of these phenomena has been linked with the presence of charged dust in the lower earth atmosphere [22] [23]. Dusty plasma physics studies the properties of heavier charged dust in presence of electrons and ions. These mixtures occur in space and astrophysical plasmas and in laboratory and technological applications. These dust grains may be negatively or positively charged depending on the dust charging mechanisms. If the dust grains are charged by electron and ion current they will be negatively charged. Positively charged dust grains may appear if any emission process e.g. photoemission process, secondary emission process is present in the environment [24]-[26].

Polar mesospheric summer echoes (PMSEs) and Noctilucent clouds (NLCs) are an intriguing example of the effects of charging of ice particles in a plasma. These are unusual atmospheric effects connected with charged ice particles in the Earth's mesosphere, at about $85 \mathrm{~km}$ altitude. The “echoes” refers to unusually strong radar backscattering which is not completely understood, but thought to be connected to electron density anomalies associated with the charged ice. Although there is no consensus on the mechanism for producing PMSEs, the current thinking is that the enhanced radar backscatter is caused by some irregularity structure in the electron density profile such as steep density gradients. Rocket measurements seem to indicate that the ice particles are 
charged positively [27], even during the night hours when the photoelectric emission of electrons from the ice is not operable. The cause of this positive charging may be due to secondary electron emission from charged ice particles. The presence of charged dust modifies the dispersion properties of plasma waves as well as their excitation conditions as detailed in references [28]-[30].

Since the dust is one of the charged fluid components of the plasma, it can support wave modes in the same way that an ordinary plasma supports ion acoustic waves. The Dust acoustic wave (DAW) is a compressional disturbance which propagates through the dust and directly involves the dynamics of the dust particles. Since the dust particles are relatively massive, DAW is a very low frequency wave, just a few $\mathrm{Hz}$ typically and propagates at a speed of a few $\mathrm{cm} / \mathrm{s}$ [31]. Effect of nonthermal ion distribution on linear and nonlinear dust acoustic wave propagation has been investigated by several authors [30] [32]-[34].

Effects of nonthermal electrons on nonlinear dust acoustic solitary waves have been investigated in presence of negatively charged dust grains [35]. Large dust acoustic solitons and kinks in dusty plasma were studied with nonthermal electrons and fixed charged positive dusts [36]. A magnetized dusty plasma consisting of positively and negatively charged adiabatic dust particles, Maxwellian ions and nonthermal electrons was considered to investigate the solitary potential structures assuming the charges on the dust grains were fixed [21].

In this paper we have developed a model to study the role of both electron and ion nonthermalities on dust acoustic wave propagation in a complex plasma in presence of positively charged dust grains. Secondary electron emission process has considered as the source of positive dust charging. Expression of nonthermal ion current in presence of positively charged dust grains was evaluated in reference [30] using OML theory [24]-[26] to study its effect on dust acoustic wave propagation. In this paper we have evaluated nonthermal primary electron current flowing to the positively charged dust grains. This consequently modifies the secondary electron current flowing out of the dust grains which has been taken into consideration in this paper. Incorporating these current expressions in the grain charging equation we have obtained the dispersion relation of dust acoustic waves. Real frequency and growth rate of the wave has been calculated from this dispersion relation. Numerical results show that when dust grains are positively charged, purely growing dust acoustic modes develop if either of electrons and ions or both are nonthermal. Growth of the instability is stronger when nonthermal electrons are present. Thus even weak electron nonthermality strongly destabilizes dust acoustic waves when equilibrium dust charge is positive.

\section{Calculation of Different Current Expressions and Primary Electron Energy}

If the secondary electron emission from dust grains is taken into account then dust grains may be positively charged for high value of the secondary electron yield which is the ratio of the emitted electrons to the incident electrons. If the background electrons are nonthermally distributed the grain charging current and consequently the secondary electron current will be modified from its expression for thermal electrons. In this section we shall evaluate the primary electron current flowing to the positively charged dust grains. The induced secondary electron current flowing out of the positively charged dust grains has also been evaluated in this paper. Non thermal ion current flowing to the positively charged dust grains were evaluated by Sarkar and Maity (2013) will be mentioned here.

\section{A. Nonthermal primary electron current and primary electron energy}

We have calculated it in this section using three dimensional equilibrium state electron velocity distribution function satisfying collisionless Vlasov equation

$$
F_{e}\left(v_{e}\right)=F_{e}\left(v_{x}, v_{y}, v_{z}\right)=\frac{n_{e 0}}{1+3 a}\left(\frac{m_{e}}{2 \pi T_{e}}\right)^{3 / 2}\left[1+4 a\left(\frac{1}{2} \frac{v_{x}^{2}}{v_{t e}^{2}}-\Phi\right)^{2}\right] \exp \left(-\frac{v_{x}^{2}+v_{y}^{2}+v_{z}^{2}}{2 v_{t e}^{2}}+\Phi\right)
$$

where $a$ is the electron nonthermal parameter, $v_{x}, v_{y}, v_{z}$ are three components of electron velocity. $v_{t e}=\sqrt{\frac{k_{B} T_{e}}{m_{e}}}$ is the electron thermal velocity, $T_{e}$ is the electron temperature, $m_{e}$ is the electron mass and $\Phi=\frac{e \phi}{k_{B} T_{e}}$, $\phi$ is the electrostatic potential and $k_{\mathrm{B}}$ is the Boltzmann constant.

Using Orbit Motion Limited (OML) theory [24] we have calculated the expression of nonthermal electron current flowing to the positively charged dust grains in the form, 


$$
I_{e}=-\pi r_{0}^{2} e \sqrt{\frac{8 T_{e}}{\pi m_{e}}} \frac{n_{e 0}}{1+3 a} \exp (\Phi)\left[\left\{\left(1+\frac{24 a}{5}\right)-16 a \Phi+4 a \Phi^{2}\right\}+\Phi_{d}\left\{\left(1+\frac{8 a}{5}\right)-\frac{8}{3} a \Phi+4 a \Phi^{2}\right\}\right]
$$

For nonthermal parameter $a=0$, this expression reduces to

$$
I_{e}=-\pi r_{0}^{2} e \sqrt{\frac{8 T_{e}}{\pi m_{e}}} n_{e} \exp (\Phi)\left(1+\Phi_{d}\right)
$$

where $\Phi_{d}=\frac{e q_{d}}{r_{0} T_{e}}$. This coincides with the expression of current for Boltzmann distributed electrons flowing to the positively charged dust grains.

In this section we have also calculated the average kinetic energy of non thermal electrons. In equilibrium ( $\Phi=0$ ) non thermal electron velocity distribution reduces to

$$
F_{e}\left(v_{e}\right)=F_{e}\left(v_{x}, v_{y}, v_{z}\right)=\frac{n_{e 0}}{1+3 a}\left(\frac{m_{e}}{2 \pi T_{e}}\right)^{3 / 2} \times\left(1+a \frac{v_{x}^{4}}{v_{t e}^{4}}\right) \exp \left(-\frac{v_{x}^{2}+v_{y}^{2}+v_{z}^{2}}{2 v_{t e}^{2}}\right)
$$

Using this distribution function we have calculated the average kinetic energy of non thermal electrons in the form,

$$
E_{a v}=\frac{3}{2}\left(\frac{1+7 a}{1+3 a}\right) K T_{e}
$$

For non thermal parameter $a=0$, this reduces to $E_{a v}=\frac{3}{2} K T_{e}$ which is the average kinetic energy for thermal electrons with 3 degrees of freedom.

\section{B. Secondary electron current when primary electrons are nonthermal}

The orbital motion limited theory based expression for secondary electron current flowing out of the positively charged dust grains in presence of nonthermal electrons will be,

$$
I_{e}^{s}=3.7 \delta_{M} \pi r_{0}^{2} e \sqrt{\frac{8 T_{e}}{\pi m_{e}}} n_{e}^{P}(a, \Phi)\left(1+\Phi_{d}\right) \exp \left(\Phi_{d}-\frac{\Phi_{d}}{\sigma_{s}}\right) F_{5, B}\left(\frac{E_{M}}{4 T_{e}}\right)
$$

where $r_{0}$ is the grain radius, $m_{e}$ is the electron mass, $T_{e}$ and $T_{s}$ are the primary and secondary electron temperatures, $\delta_{M}$ is the maximum yield of secondary electrons, which occurs when the impinging electron has the maximum kinetic energy $E_{M}$. Here $n_{e}^{P}(a, \Phi)$ is the number density of nonthermal primary electrons with nonthermality parameter $a$. The function $F_{5, B}(x)$ is given by,

$$
F_{5, B}(x)=x^{2} \int_{B}^{\infty} u^{5} \exp \left[-\left(x u^{2}+u\right)\right] \mathrm{d} u
$$

where

$$
x=\frac{E_{M}}{4 T_{e}}, B=\sqrt{\frac{\frac{e q_{d}}{r_{0} T_{e}}}{x}}, \sigma_{s}=\frac{T_{s}}{T_{e}}
$$

Putting $a=0$ in the expression (6) we get back the expression of secondary electron current for thermally distributed primary electrons.

\section{Nonthermal ion current}

The OML theory based expression for the nonthermal ion current $I_{i}$ is [31],

$$
I_{i}=\pi r_{0}^{2} e \sqrt{\frac{8 T_{i}}{\pi m_{i}}} \frac{n_{i 0}}{1+3 b} \exp \left(-\frac{\Phi}{\sigma_{i}}\right)\left[\left(1+\frac{24 b}{5}\right)-\frac{8 b}{3} \frac{\Phi}{\sigma_{i}}\left(2+\frac{\Phi_{d}}{\sigma_{i}}\right)+\frac{4 b}{5}\left(5 \frac{\Phi^{2}}{\sigma_{i}^{2}}+\frac{\Phi_{d}^{2}}{\sigma_{i}^{2}}\right)+\frac{16 b}{5} \frac{\Phi_{d}}{\sigma_{i}}\right] \exp \left(-\frac{\Phi_{d}}{\sigma_{i}}\right)
$$

where $b$ is the ion nonthermal parameter [30] and $\sigma_{i}=\frac{T_{i}}{T_{e}}$. 
This was evaluated using ion velocity distribution [15]

$$
F_{i}\left(v_{i}\right)=F_{i}\left(v_{x}, v_{y}, v_{z}\right)=\frac{n_{i 0}}{1+3 b}\left(\frac{1}{2 \pi v_{t i}^{2}}\right)^{3 / 2}\left[1+4 b\left(\frac{1}{2} \frac{v_{x}^{2}}{v_{t i}^{2}}+\frac{\Phi}{\sigma_{i}}\right)^{2}\right] \exp \left(-\frac{v_{x}^{2}+v_{y}^{2}+v_{z}^{2}}{2 v_{t i}^{2}}-\frac{\Phi}{\sigma_{i}}\right)
$$

\section{Grain Charge Fluctuation: Charging Frequency}

Since number of charges on the dust grains fluctuates in space and time, dust charge is a dynamical variable satisfying the grain charging equation

$$
\frac{\partial q_{d}}{\partial t}+v_{d} \frac{\partial q_{d}}{\partial x}=I_{e}+I_{i}+I_{e}^{s}=I_{\text {total }}
$$

In this section we substitute the expressions of $I_{i}, I_{e}$ and $I_{e}^{s}$ from (2), (6) and (9) in this dust charging equation.

In equilibrium $I_{\text {total }}=0$, at $\left.q_{d}\right|_{e q}=q_{d 0}=Z_{d 0} e$ (positively charged dust) $\left.n_{i}\right|_{\phi=0}=n_{i 0} ;\left.n_{e}\right|_{\phi=0}=n_{e 0}$ when

$$
\Phi=0
$$

From (10) and (11) with (2), (6) and (9) we calculate the equilibrium ion-electron number density ratio in the form,

$$
\frac{n_{i 0}}{n_{e 0}}=\sqrt{\frac{m_{i}}{m_{e}}} \exp \left(\frac{z_{0}}{\sigma_{i}}\right) \frac{1}{\sqrt{\sigma_{i}}} \frac{1+3 b}{\left(1+\frac{24 b}{5}+\frac{16 b}{5} \frac{z_{0}}{\sigma_{i}}+\frac{4 b}{5} \frac{z_{0}^{2}}{\sigma_{i}^{2}}\right)} \times \alpha_{1 s}^{+}
$$

where $z_{0}=\frac{z_{d 0} e^{2}}{r_{0} T_{e}}$ and

$$
\alpha_{1 s}^{+}=\frac{5+24 a}{5(1+3 a)}+z_{0} \frac{5+8 a}{5(1+3 a)}-3.7 \delta_{M}\left(1+z_{0}\right) \exp \left(z_{0}-\frac{z_{0}}{\sigma_{s}}\right) F_{5, B}\left(\frac{E_{M}}{4 T_{e}}\right)
$$

The grain charging frequency for positively charged dust grains in the background of nonthermal electrons, ions and with emitted secondary electrons has been calculated in the form,

$$
v_{c h}^{+}=\frac{\frac{r_{0}}{\lambda_{D_{i}}}}{\sqrt{2 \pi}} \omega_{p_{i}} \exp \left(-\frac{z_{0}}{\sigma_{i}}\right)\left[\beta+B_{10} \sigma_{i} \frac{\alpha_{2 s}^{+}}{\alpha_{1 s}^{+}}\right]
$$

where

$$
\begin{aligned}
& \beta=\frac{1+\frac{8 b}{5}+\frac{8 b}{5} \frac{z_{0}}{\sigma_{i}}+\frac{4 b}{5} \frac{z_{0}^{2}}{\sigma_{i}^{2}}}{1+3 b}, B_{10}=\frac{1+\frac{24 b}{5}+\frac{16}{b} \frac{z_{0}}{\sigma_{i}}+\frac{4 b}{5} \frac{z_{0}^{2}}{\sigma_{i}^{2}}}{1+3 b} \\
& \alpha_{1 s}^{+}=\frac{5+24 a}{5(1+3 a)}+z_{0} \frac{5+8 a}{5(1+3 a)}-3.7 \delta_{M}\left(1+z_{0}\right) \exp \left(z_{0}-\frac{z_{0}}{\sigma_{s}}\right) F_{5, B}\left(\frac{E_{M}}{4 T_{e}}\right) \\
& \alpha_{2 s}^{+}=\frac{5+8 a}{5(1+3 a)}-3.7 \delta_{u} \exp \left(z_{0}-\frac{z_{0}}{\sigma_{s}}\right) F_{5, B_{0}}\left(\frac{E_{M}}{4 T_{e}}\right) \times\left[1+\left(1+z_{0}\right)\left\{1-\frac{1}{\sigma_{s}}-\frac{1}{2} \frac{z_{0}^{2} \exp \left(-z_{0}-\sqrt{\frac{z_{0}}{E_{M} / 4 T_{e}}}\right)}{\left(\frac{E_{M}}{4 T_{e}}\right) F_{5, B_{0}}\left(\frac{E_{M}}{4 T_{e}}\right)}\right\}\right] \\
& \sigma_{s}=\frac{T_{s}}{T_{e}}, \quad \lambda_{D_{i}}=\frac{T_{i}}{4 \pi n_{i 0} e^{2}}, \omega_{p_{i}}=\sqrt{\frac{4 \pi n_{i 0} e^{2}}{m_{i}}}
\end{aligned}
$$

Linearization of Equations (10) with (2), (6), (9) and (11) leads to the linearized charge variation, 


$$
q_{1}=-\frac{\beta_{d}}{v_{c h}^{+}-i \omega} \Phi
$$

where

$$
\beta_{d}=\frac{r_{0} / \lambda_{D_{i}}}{\sqrt{2 \pi}} \omega_{p_{i}} \exp \left(-\frac{z_{0}}{\sigma_{i}}\right) \frac{\sigma_{i}}{z_{0}} \frac{\left(1+\frac{24 b}{5}+\frac{16 b}{5} \frac{z_{0}}{\sigma_{i}}+\frac{4 b}{5} \frac{z_{0}^{2}}{\sigma_{i}^{2}}\right)}{1+3 b} \times\left(\frac{1-b}{1+3 b} \cdot \frac{1}{\sigma_{i}}+\frac{1-a}{1+3 a}\right)
$$

\section{Governing Equations and Dispersion Relation}

We have considered a plasma consisting of non thermal electrons, ions and positively charged inertial dust grains satisfying the equilibrium charge neutrality condition,

$$
n_{i 0}-n_{e 0}-n_{e 0}^{s}+z_{d 0} n_{d 0}=0
$$

The governing equations are

$$
\begin{gathered}
n_{e}^{P}(a, \Phi)=n_{e 0}\left[1+\frac{4 a}{1+3 a}\left(-\Phi+\Phi^{2}\right)\right] \exp (\Phi) \\
n_{e}^{s}(a, \Phi)=n_{e 0}\left[1+\frac{4 a}{1+3 a}\left(-\frac{\Phi}{\sigma_{s}}+\frac{\Phi^{2}}{\sigma_{s}^{2}}\right)\right] \exp \left(\frac{\Phi}{\sigma_{s}}\right) \\
n_{i}(b, \Phi)=n_{i 0}\left[1+\frac{4 b}{1+3 b}\left(\frac{\Phi}{\sigma_{i}}+\frac{\Phi^{2}}{\sigma_{i}^{2}}\right)\right] \exp \left(-\frac{\Phi}{\sigma_{i}}\right) \\
\frac{\partial n_{d}}{\partial t}+\frac{\partial}{\partial x}\left(n_{d} v_{d}\right)=0 \\
\frac{\partial v_{d}}{\partial t}+v_{d} \frac{\partial v_{d}}{\partial x}=-\frac{q_{d}}{m_{d}} \frac{\partial \phi}{\partial x}-\frac{T_{d}}{m_{d} n_{d}} \frac{\partial n_{d}}{\partial x} \\
\frac{\partial q_{d}}{\partial t}+v_{d} \frac{\partial q_{d}}{\partial x}=I_{e}+I_{i}+I_{e}^{s}
\end{gathered}
$$

and the Poisson equation

$$
\frac{\partial^{2} \phi}{\partial x^{2}}=-4 \pi\left(e n_{i}-e n_{e}^{P}-e n_{e}^{s}+q_{d} n_{d}\right)
$$

Here $m_{d}$ is the mass of the charged dust grains moving with velocity $v_{d}$ and $n_{d}$ is the dust number density.

Linearizing these basic equations about their equilibrium values we obtain the first order perturbed number densities of primary electrons, secondary electrons, ions and dust grains in the form,

$$
\partial n_{e}^{P}=n_{e 0} \frac{1-a}{1+3 a} \Phi, \partial n_{e}^{s}=n_{e 0} \frac{1-a}{1+3 a} \frac{\Phi}{\sigma_{s}}, \partial n_{i}=-n_{i 0} \frac{1-b}{1+3 b} \frac{\Phi}{\sigma_{i}}, \partial n_{d}=n_{d 0} \frac{k^{2} C_{d a}^{2}}{\omega^{2}-k^{2} v_{t d}^{2}} \Phi
$$

where

$$
C_{d a}^{2}=\frac{Z_{d 0} T_{e}}{m_{d}}
$$

Substituting $\partial n_{e}^{P}, \partial n_{e}^{S}, \partial n_{i}, \partial n_{d}$ from (25) and $q_{i}$ from (16) in the linearized poisson equation we obtain the following dispersion relation for dust acoustic waves in the long wave length approximation,

$$
\frac{1-a}{1+3 a}+\frac{1-a}{1+3 a} \cdot \frac{1}{\sigma_{s}}+\frac{1-b}{1+3 b} \frac{1}{\sigma_{i}} \frac{n_{i 0}}{n_{e 0}}+P \frac{\beta_{d}}{v_{c h}^{+}-i \omega}-\frac{P k^{2} C_{d a}^{2}}{\omega^{2}-k^{2} v_{t d}^{2}}=0
$$

where $P=\frac{n_{d_{0}} Z_{d_{0}}}{n_{e_{0}}}$. 
From this dispersion relation we have calculated the real frequency $\omega_{r}$ and growth rate $\omega_{i}$ in the form,

$$
\begin{gathered}
\frac{\omega_{r}^{2}}{k^{2} C_{d a}^{2}}=\sigma_{d}+\frac{\frac{Z_{d 0} n_{d 0}}{n_{e 0}}}{\frac{1-a}{1+3 a}+\frac{1-a}{1+3 a} \cdot \frac{1}{\sigma_{s}}+\frac{1-b}{1+3 b} \cdot \frac{1}{\sigma_{i}} \frac{n_{i 0}}{n_{e 0}}+\frac{Z_{d 0} n_{d 0}}{n_{e 0}} \frac{\beta_{d}}{v_{c h}^{+}}} \\
\frac{\omega_{v_{c h}}^{+}}{k^{2} C_{d a}^{2}}=-\frac{1}{2}\left(\frac{\beta_{d}}{v_{c h}^{+}}\right) \times\left[\begin{array}{c}
\frac{1}{\frac{1-a}{1+3 a}+\frac{1-a}{1+3 a} \cdot \frac{1}{\sigma_{s}}+\frac{1-b}{1+3 b} \cdot \frac{1}{\sigma_{i}} \frac{n_{i 0}}{n_{e 0}}}+\frac{\beta_{d}}{V_{c h}^{+} n_{d 0}} \\
n_{e 0}
\end{array}\right]
\end{gathered}
$$

\section{Numerical Estimation}

For numerical estimation we have considered that dust grains are MgO material having $\delta_{M} \sim 22-24$ and $E_{M}(\mathrm{eV}) \sim 350-1500$. Graphs of $\omega_{r}^{2}$ and $\omega_{i}$ have plotted against $z_{0}$ for four sets of values of $a$ and $b$ with $\sigma_{i}$ $=1.0, \sigma_{s}=1.01$ and $\delta_{M}=24$. Figure 1 and Figure 2 have been plotted for $\omega_{r}^{2}$ and $\omega_{i}$ against $z_{0}$ for $a=0.05$ and $b=0.5$. The same have been plotted for $a=0.05, b=0.0$ in Figure 3 and Figure 4, $a=0.0, b=1.5$ in Figure 5 and Figure 6 and $a=0.0, b=0.0$ in Figure 7 and Figure 8. In first three cases $\omega_{r}^{2}<0$ implies $\omega_{r}$ is imaginary. Thus growth or decay of the wave will depend on the sign of $\left|\omega_{r}\right|+\omega_{i}$. In the fourth case when electrons and ions are both thermal $\omega_{r}^{2}>0$. Hence $\omega_{r}$ is real. Thus growth or decay of the wave in this case will depend only on the sign of $\omega_{i}$. Figure 2, Figure 4 and Figure 6 show that $\omega_{i}$ is positive and increases with increasing $z_{0}$ whereas Figure 8 shows that $\omega_{i}$ is negative and its magnitude increases with increasing $z_{0}$. Figures 9-12 have been plotted for $\left|\omega_{r}\right|+\omega_{i}$ versus $z_{0}$ with the values $(a=0.05, b=0.5),(a=0.05, b=0.0),(a$ $=0.0, b=1.5)$, and $(a=0.0, b=0.0)$ respectively.

In Figures 9-11, $\left|\omega_{r}\right|+\omega_{i}$ is positive and increases with increasing values of $z_{0}$. So dust acoustic wave grows when either of electrons and ions or both are nonthermal. In all the three cases, magnitude of growth rate increases with increasing number of charges on the positively charged dust grains. Magnitude of growth is stronger in first two cases when electrons are nonthermal but rate of increase of the growth with $z_{0}$ in these cases are slower than the case when electrons are thermal but ions are nonthermal. Figure 12 shows that dust acoustic wave decays when electrons and ions are both thermal even in presence of positively charged dust grains.

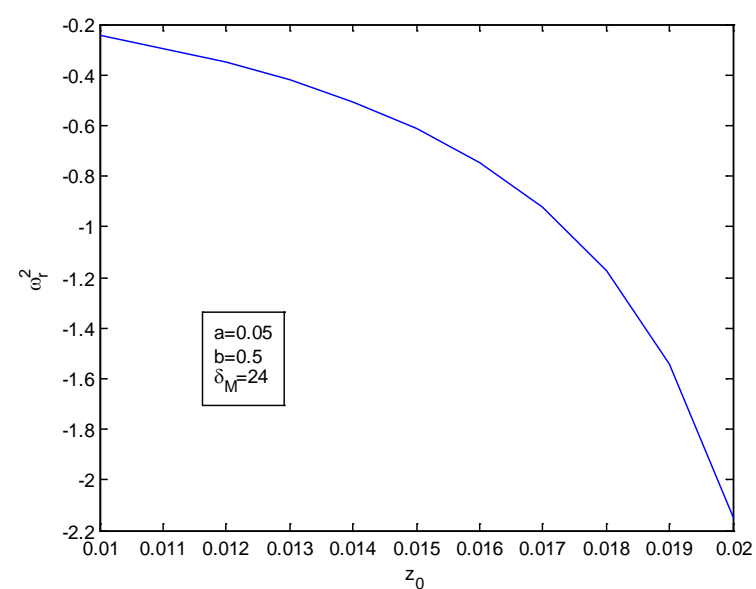

Figure 1. Plot of the squared real frequency $\omega_{r}^{2}$ vs $z_{0}$ for positively charged dust grains when nonthermal parameters $a=0.05$ and $b=0.5$. 


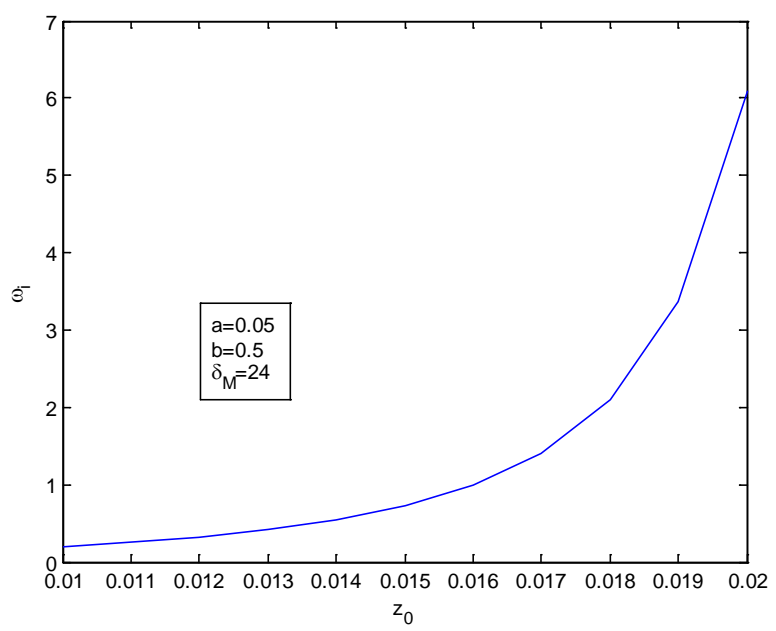

Figure 2. Plot of the imaginary frequency $\omega_{i}$ vs $z_{0}$ for positively charged dust grains when nonthermal parameters $a=0.05$ and $b=0.5$.

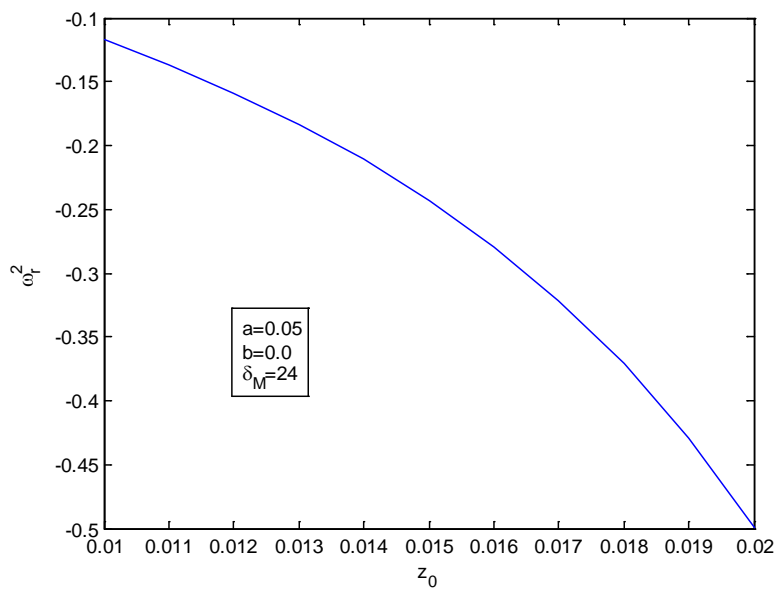

Figure 3. Plot of the squared real frequency $\omega_{r}^{2}$ vs $z_{0}$ for positively charged dust grains when nonthermal parameters $a=0.05$ and $b=0.0$.

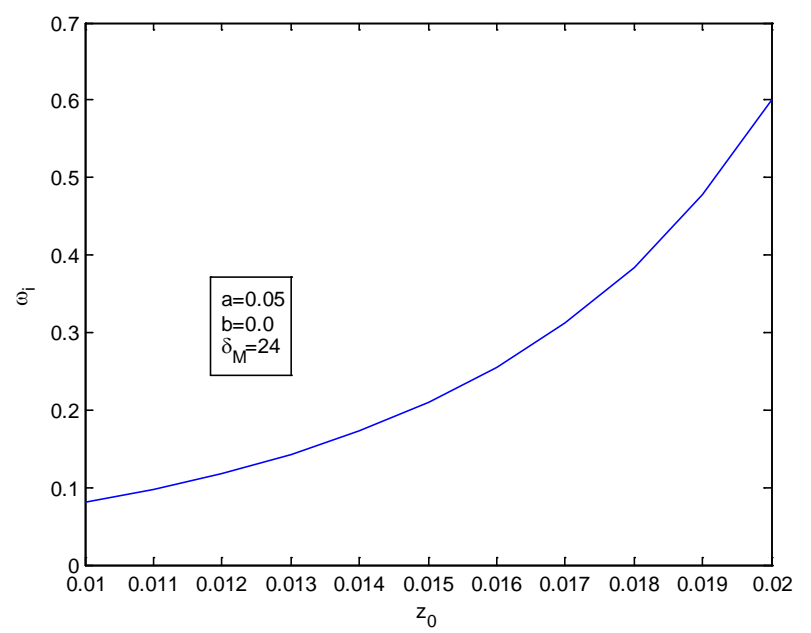

Figure 4. Plot of the imaginary frequency $\omega_{i}$ vs $z_{0}$ for positively charged dust grains when nonthermal parameters $a=0.05$ and $b=0.0$. 


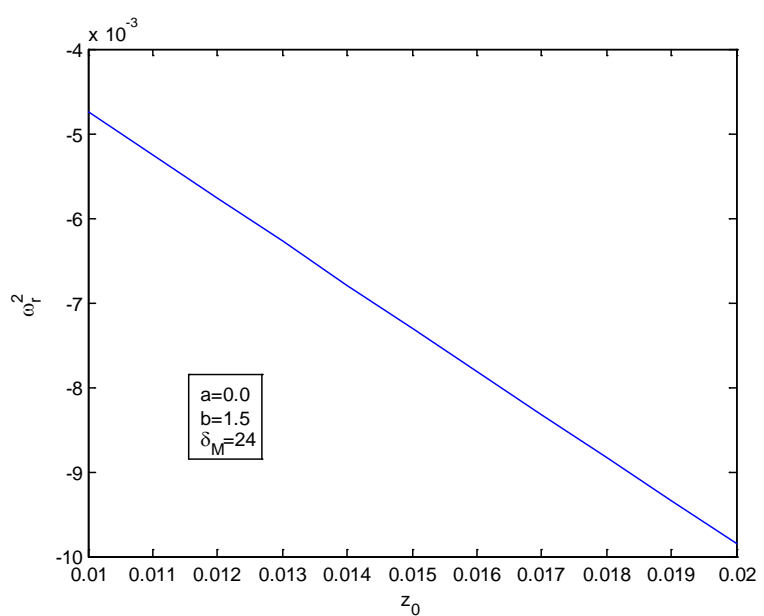

Figure 5. Plot of the squared real frequency $\omega_{r}^{2}$ vs $z_{0}$ for positively charged dust grains when nonthermal parameters $a=0.0$ and $b=1.5$.

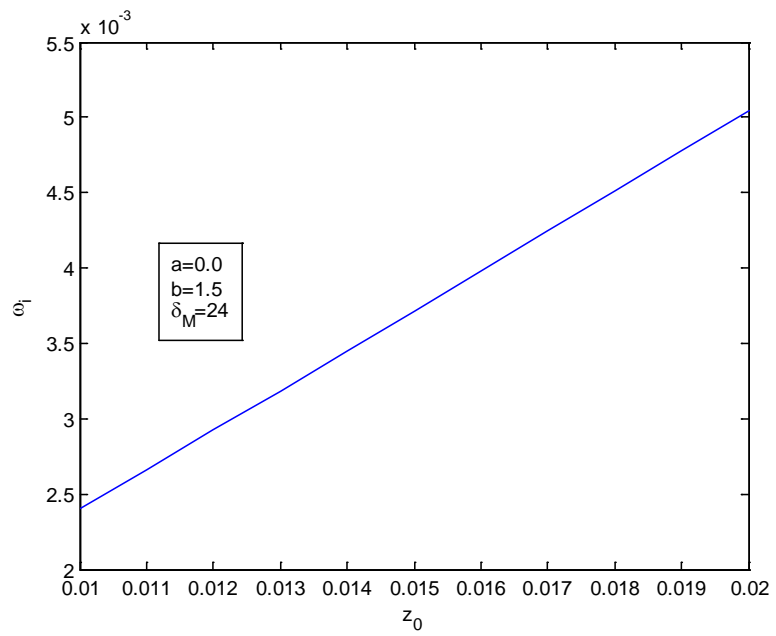

Figure 6. Plot of the imaginary frequency $\omega_{i}$ vs $z_{0}$ for positively charged dust grains when nonthermal parameters $a=0.0$ and $b=1.5$.

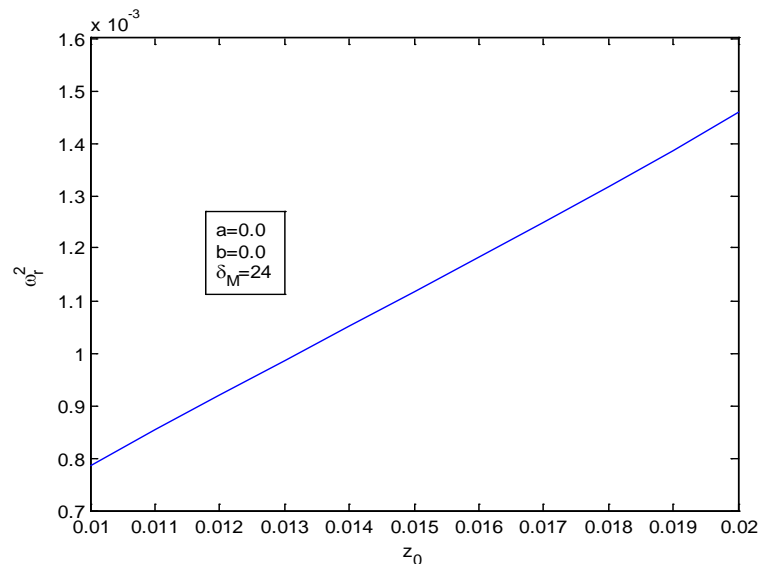

Figure 7. Plot of the squared real frequency $\omega_{r}^{2}$ vs $z_{0}$ for positively charged dust grains when nonthermal parameters $a=0.0$ and $b=0.0$. 


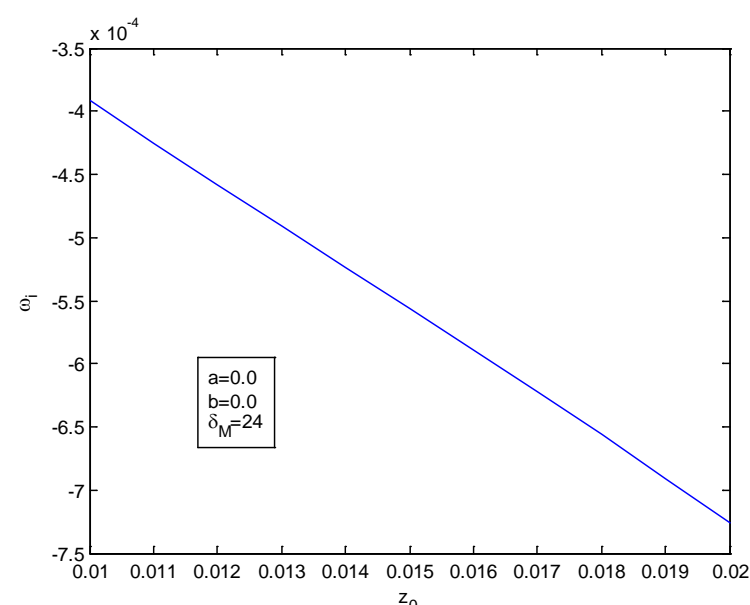

Figure 8. Plot of the imaginary frequency $\omega_{i}$ vs $z_{0}$ for positively charged dust grains when nonthermal parameters $a=0.0$ and $b=0.0$.

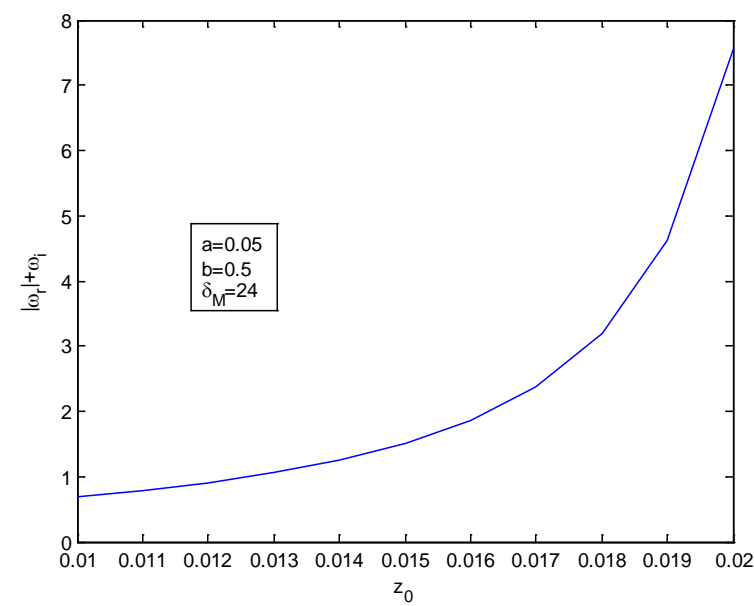

Figure 9. Plot of $\left(\left|\omega_{r}\right|+\omega_{i}\right)$ vs $z_{0}$ for positively charged dust grains when nonthermal parameters $a=0.05$ and $b=0.5$.

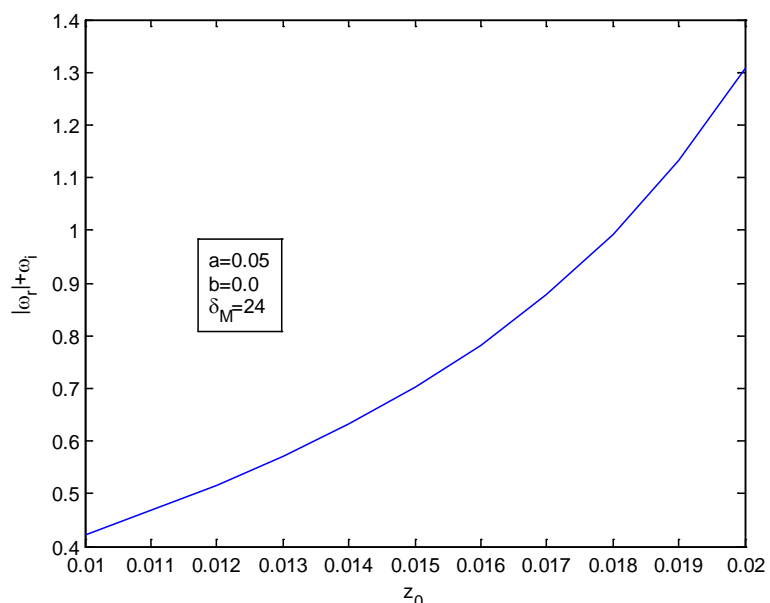

Figure 10. Plot of $\left(\left|\omega_{r}\right|+\omega_{i}\right)$ vs $z_{0}$ for positively charged dust grains when nonthermal parameters $a=0.05$ and $b=0.0$. 


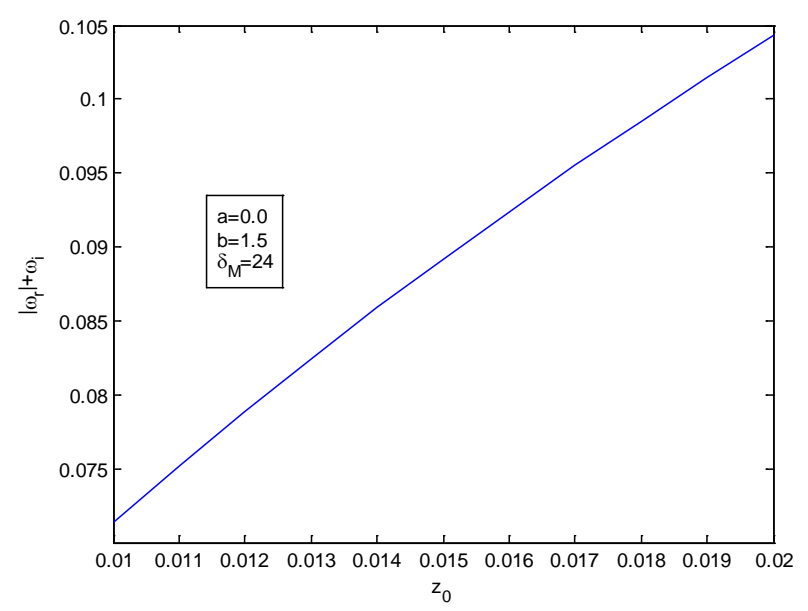

Figure 11. Plot of $\left(\left|\omega_{r}\right|+\omega_{i}\right)$ vs $z_{0}$ for positively charged dust grains when nonthermal parameters $a=0.0$ and $b=1.5$.

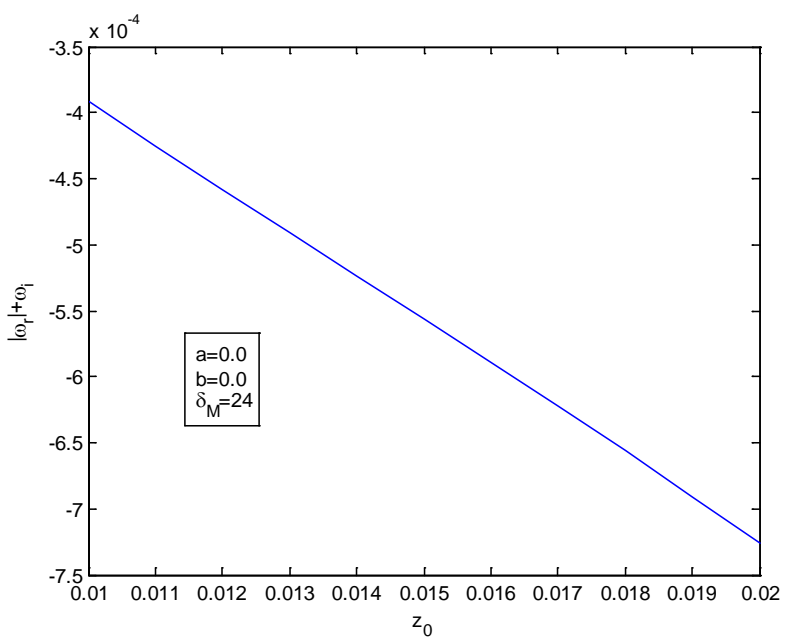

Figure 12. Plot of $\left(\left|\omega_{r}\right|+\omega_{i}\right)$ vs $z_{0}$ for positively charged dust grains when nonthermal parameters $a=0.0$ and $b=0.0$.

\section{Conclusion}

The effect of both electron and ion nonthermalities on dust acoustic wave propagation in a complex plasma in presence of positively charged dust grains has been investigated in this paper. Secondary electron emission has been considered as the source of positive dust charging. This secondary electron emission process is influenced by the nonthermality of primary electrons hitting the dust grains. Dispersion relation of dust acoustic wave has been obtained with the above mentioned physical effects. From the dispersion relation we have calculated the real frequency $\omega_{r}$ and growth rate $\omega_{i}$ of the wave. Numerically we have shown that in presence of positively charged dust grains purely growing dust acoustic modes develop when either of electrons and ions or both are nonthermal and magnitude of the growth rate increases with increasing number of charges on the dust grains. Moreover growth rate is stronger when electrons are nonthermal. Thus even weak electron nonthermality strongly destabilizes dust acoustic waves when equilibrium dust charge is positive.

\section{References}

[1] Anderson, K.A., Harris, H.K. and Paoli, R.J. (1965) Journal of Geophysical Research, 70, 1039-1050. http://dx.doi.org/10.1029/JZ070i005p01039 
[2] Gosling, J.T., et al. (1989) Journal of Geophysical Research, 94, 10011-10025.

[3] Krauss-Varban, D.J. (1994) Journal of Geophysical Research: Space Physics, 99, 2537-2551. http://dx.doi.org/10.1029/93JA01643

[4] Oka, M., Terasawa, T., Kasaba, Y., Kojima, H., Fujimoto, M., Matsumoto, H., Saito, Y. and Mukai, T. (2005) Proceedings of ISSS, 7, 26-31.

[5] Emslie, A.G., Phillips, K. and Denis, B. (1986) Solar Physics, 103, 89-102. http://dx.doi.org/10.1007/BF00154860

[6] Micela, G., Favata, F., Giardino, G. and Sciortino, S. (2008) Mem.S.A.It, 79, 264.

[7] Oka, M., Terasawa, T., Fujimoto, M., Matsui, H., Kasaba, Y., Saito, Y., Kojima, H., Matsumoto, H. and Mukai, T. (2009) Earth, Planets and Space, 61, 603-606. http://dx.doi.org/10.1186/BF03352932

[8] Eliasson, B., Hirth, M. and Kogelschatz, U. (1987) Journal of Physics D: Applied Physics, 20, 1421-1437. http://dx.doi.org/10.1088/0022-3727/20/11/010

[9] Kogelschatz, U. (2003) Plasma Chemistry and Plasma Processing, 23, 1-46. http://dx.doi.org/10.1023/A:1022470901385

[10] Lister, G., Lawler, J., Lapatovich, W. and Godyak, V. (2004) Reviews of Modern Physics, 76, 541-598. http://dx.doi.org/10.1103/RevModPhys.76.541

[11] Ehlbeck, J., Schnabel, U., Polak, M., Winter, J., von Woedtke, T., Brandenburg, R., von dem Hagen, T. and Weltmann, K.-D. (2011) Journal of Physics D: Applied Physics, 44, Article ID: 013002. http://dx.doi.org/10.1088/0022-3727/44/1/013002

[12] Becker, M.M. and Loffhagen, D. (2013) Advances in Pure Mathematics, 3, 343-352. http://dx.doi.org/10.4236/apm.2013.33049

[13] Bostrom, R. (1992) IEEE Transactions on Plasma Science, 20, 756-763. http://dx.doi.org/10.1109/27.199524

[14] Dovner, P.O., Eriksson, A.I., Bostrom, R. and Holback, B. (1994) Geophysical Research Letters, 21, 1827-1830. http://dx.doi.org/10.1029/94GL00886

[15] Cairns, R.A., Bingham, R., Dendy, R.O., Nairn, C.M.C., Shukla, P.K. and Mamun, A.A. (1995) Journal de Physique IV, 5, C6-C43.

[16] Cairns, R.A., Mamun, A.A., Bingham, R., Bostrom, R., Dendy, R.O., Nairn, C.M.C. and Shukla, P.K. (1995) Geophysical Research Letters, 22, 2709-2712. http://dx.doi.org/10.1029/95GL02781

[17] Mamun, A.A. (1997) Physical Review E, 55, 1852-1857. http://dx.doi.org/10.1103/PhysRevE.55.1852

[18] Mamun, A.A. (2000) The European Physical Journal D-Atomic, Molecular, Optical and Plasma Physics, 11, 143147. http://dx.doi.org/10.1007/s100530070115

[19] Tang, R.-A. and Xue, J.-K. (2004) Physics of Plasma, 11, 3939-3944.

[20] Verheest, F., Hellberg, M.A. and Hereman, W.A. (2012) Physical Review E, 86, Article ID: 036402. http://dx.doi.org/10.1103/PhysRevE.86.036402

[21] Kakati, B., Kausik, S.S., Saikia, B.K. and Bandyopadhyay, M. (2011) Physics of Plasmas, 18, Article ID: 033705. http://dx.doi.org/10.1063/1.3568841

[22] Gelinas, L.J., Lynch, K.A., Kelley, M.C., Collins, S., Baker, S., Zhou, Q. and Friedman, J.S. (1998) Geophysical Research Letters, 25, 4047-4050. http://dx.doi.org/10.1029/1998GL900089

[23] Gelinas, L.J. (1999) An in Situ Measurement of Charged Mesospheric Dust during a Sporadic Atom Layer Event. PhD Thesis, University of New Hampshire, Durham.

[24] Meyer-Vernet, N. (1982) Astronomy \& Astrophysics, 105, 98-106.

[25] Horyani, M., Roberston, S. and Walch, B. (1995) Geophysical Research Letters, 22, 2079-2082. http://dx.doi.org/10.1029/95GL02287

[26] Goertz, C.K. (1989) Reviews of Geophysics, 27, 271-292. http://dx.doi.org/10.1029/RG027i002p00271

[27] Rapp, M., Hedin, J., Strelnikova, I., Friedrich, M., Gumbel, J. and Lübken, F.-J. (2005) Geophysical Research Letters, 32, Article ID: L23821. http://dx.doi.org/10.1029/2005GL024676

[28] Shukla, P.K. and Mamun, A.A. (2002) Introduction to Dusty Plasma Physics. IOP, Bristol.

[29] Merlino, R.L. (2006) Plasma Physics Applied, 81, 73-110.

[30] Verheest, F. (1996) Space Science Reviews, 77, 267-302.

[31] Sarkar, S., Maity, S. and Banerjee, S. (2011) Physica Scripta, 84, Article ID: 045501. http://dx.doi.org/10.1088/0031-8949/84/04/045501

[32] Sarkar, S. and Maity, S. (2013) Physics of Plasmas, 20, Article ID: 084501. http://dx.doi.org/10.1063/1.4817739 
[33] Ghosh, S., Bharuthram, R., Khan, M. and Gupta, M.R. (2004) Physics of Plasmas, 11, 3602. http://dx.doi.org/10.1063/1.1760584

[34] Mamun, A.A. and Shukla, P.K. (2009) Physical Review E, 80, Article ID: 037401. http://dx.doi.org/10.1103/PhysRevE.80.037401

[35] Verheest, F. and Pillai, S.R. (2008) Nonlinear Processes in Geophysics, 15, 551-555. http://dx.doi.org/10.5194/npg-15-551-2008

[36] Bedi, C., Gill, T.S. and Bains, A.S. (2010) Journal of Physics: Conference Series, 208, Article ID: 012037. http://dx.doi.org/10.1088/1742-6596/208/1/012037 